\title{
Ubicación de la Zona de Inervación del Músculo Flexor Corto del Pulgar en Individuos Sanos de Ambos Sexos
}

\author{
Flexor Pollicis Brevis Muscle Innervation Zone Location in Healthy Individuals of Both Sexes
}

\author{
Rodrigo A. Guzmán; María Pilar Bralic Echeverria \& José Cordero Garayar
}

GUZMÁN, R. A.; BRALIC, E. M. P. \& CORDERO, G. J. Ubicación de la zona de inervación del músculo flexor corto del pulgar en individuos sanos de ambos sexos. Int. J. Morphol., 31(2):449-454, 2013.

RESUMEN: El músculo flexor corto del pulgar (FCP) se localiza en la eminencia tenar y tiene un importante rol en las funciones del dedo pulgar. Existen escasos antecedentes acerca de la distribución de las zonas de inervación (ZI) de este músculo y las posibles diferencias entre individuos de diferente sexo. El conocimiento de la localización de las ZI del FCP podría ser de ayuda en el tratamiento de la mano espástica, sirviendo para definir el sitio de inyección de la toxina botulínica. El objetivo de esta investigación fue describir las ubicaciones de las ZI del FCP usando electromiografía de superficie, y hacer una comparación entre individuos de sexo masculino y femenino. Treinta jóvenes voluntarios sanos, participaron en este estudio ( 15 hombres: $21.5 \pm 2.6$ años, $70.7 \pm 7.2 \mathrm{~kg}$ y $175.0 \pm 5.5 \mathrm{~cm} .15$ Mujeres: $19.9 \pm 1.4$ años, $57.9 \pm 11.1 \mathrm{~kg}$ y $161.9 \pm 6.6 \mathrm{~cm})$. Las ZI fueron identificadas mediante la grabación de los potenciales de acción de las unidades motoras del FCP, usando un arreglo de dieciséis electrodos de superficie. Los potenciales fueron grabados durante contracciones isométricas al 10\% de la contracción voluntaria máxima. Las localizaciones de las ZI fueron expresadas en forma absoluta y relativa en relación a un sistema de referencia construido en la palma de la mano, en base a referencias anatómicas. No existieron diferencias significativas en las ubicaciones de las ZI entre los participantes de sexo masculino y femenino de la muestra evaluada. Para el grupo de participantes en este estudio, las ZI del FCP se encontraron al $41.9 \%$ de la distancia comprendida entre el extremo palpable más distal y medial de la interlínea articular metacarpofalángica del pulgar y la línea que nace en el vértice palpable del extremo proximal de la primera falange del tercer dedo pasado por el pliegue longitudinal radial.

PALABRAS CLAVE: Unidad Motora; Zona de Inervación; Punto motor; Flexor corto del pulgar.

\section{INTRODUCCIÓN}

El músculo flexor corto del pulgar (FCP) forma parte de los músculos de la eminencia tenar. Su acción es importante en la función motora de la mano, dado que es sinergista del músculo oponente del pulgar. Ambos participan en la ejecución de la pinza, la cual es una tarea motora esencial de la mano. (Gupta \& Michelsen-Jost, 2012). El FCP presenta dos porciones; una superficial y otra profunda, que son inervadas por el nervio mediano y ulnar, respectivamente. (Gupta \& Michelsen-Jost). La porción superficial de este músculo puede ser estudiada mediante electromiografía de superficie (Rainoldi et al., 2008; Danion \& Gallea, 2004). La contracción del FCP mueve el pulgar en el plano de la palma sobre la articulación metacarpofalángica y puede producir una ligera flexión de la carpometacarpiana (Gupta \& Michelsen-Jost).

La función motora de la mano determina en gran medida la funcionalidad e independencia de una persona en las tareas motoras cotidianas. A consecuencia de lesiones neurológicas congénitas o adquiridas, la función de la mano puede verse gravemente afectada. En este sentido, uno de los fenómenos que mayormente aqueja a los pacientes con daño neurológico es la espasticidad. Cuando ésta se localiza en la musculatura de la mano, deteriora en forma muy importante su función motora. Una de las alternativas terapéuticas para tratar la espasticidad y mejorar la función de músculos espásticos es la aplicación de toxina botulínica (TB), la cual tiene por acción evitar la liberación de acetilcolina en la placa motora. Esta última estructura topográficamente corresponde a la zona de inervación (ZI). (Saitou et al., 2000). El bloqueo de la liberación de acetilcolina tiene por objetivo disminuir el tono muscular, aumentado a consecuencia de la espasticidad. La eficacia de la aplicación de la TB ha demostrado ser sensible a la distancia entre el sitio de inyección de ésta con respecto a la ZI. (Lapatki et al., 2011). En nuestros conocimientos, no 
existen reportes que describan la ubicación de la ZI en el FCP y las posibles diferencias entre sujetos de distinto sexo. Contar con datos acerca de la ubicación y distribución de las zonas de inervación del músculo FCP, podría entregar información de consideración en la aplicación de la TB en el marco del tratamiento de la mano espástica de pacientes con lesiones neurológicas. El objetivo principal de este estudio fue describir la ubicación de la ZI del músculo FCP en una muestra de voluntarios sanos, utilizando electromiografía de superficie de alta densidad. Como objetivo secundario, se buscó describir la influencia del sexo en ubicación de la ZI del FCP.

\section{MATERIAL Y MÉTODO}

Diseño. Esta investigación responde a un modelo descriptivo analítico de corte transversal.

Participantes. Participaron 30 voluntarios, distribuidos en 15 mujeres y 15 hombres, todos diestros. Los participantes fueron reclutados mediante un muestreo por conveniencia, según los siguientes criterios de exclusión: (1) Dominancia manual izquierda; (2) Historial de lesión musculoesquelética de extremidad superior. (3) Patologías neurológicas centrales o periféricas. (4) Lesiones cutáneas en la zona de registro. (5) Práctica deportiva que involucre hipertrofia de los músculos de la mano (Tenis, Tenis de mesa, Judo, etc).

Todos los participantes fueron informados de manera verbal de los procedimientos y objetivos del estudio. Además se les solicitó consentimiento informado. Los procedimientos realizados en este estudio fueron aprobados por el comité de Bioética de la Facultad de Medicina de la Universidad de los Andes.

Instrumentación. Para la ubicación de la ZI, se registraron los potenciales de acción de las unidades motoras (PAUM) del FCP, utilizando una técnica de electromiografía de superficie (EMGs). Para ello se utilizó un arreglo lineal de 16 electrodos de superficie, construidos en plata al 99\%. Con una distancia interelectrodos de $2,5 \mathrm{~mm}$ (SA 16/2.5 OTbiolettronica. Torino Italia), tal como se puede apreciar en la Figura 1. Las señales EMGs, fueron amplificadas con una ganancia de 2000 unidades, filtradas en un ancho de banda de $10-500 \mathrm{~Hz}$ y digitalizadas a $2048 \mathrm{~Hz}$ con una resolución de 12 bits, todo mediante un amplificador de señales biomédicas (EMG-USB2. OTbiolettronica. Torino. Italia).

Se utilizó un sensor de fuerza (DAQ3. Kinetecnics. Santiago, Chile) para valorar y controlar la fuerza generada en el sentido de la flexión del pulgar. Dicho sensor fue ubi-

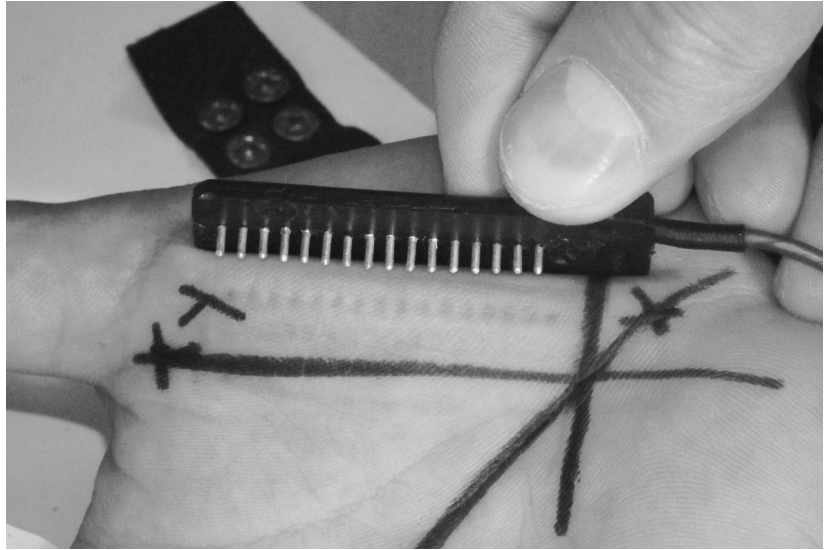

Fig. 1. Arreglo de 16 electrodos de superficie con una distancia interelectrodo de $2.5 \mathrm{~mm}$.

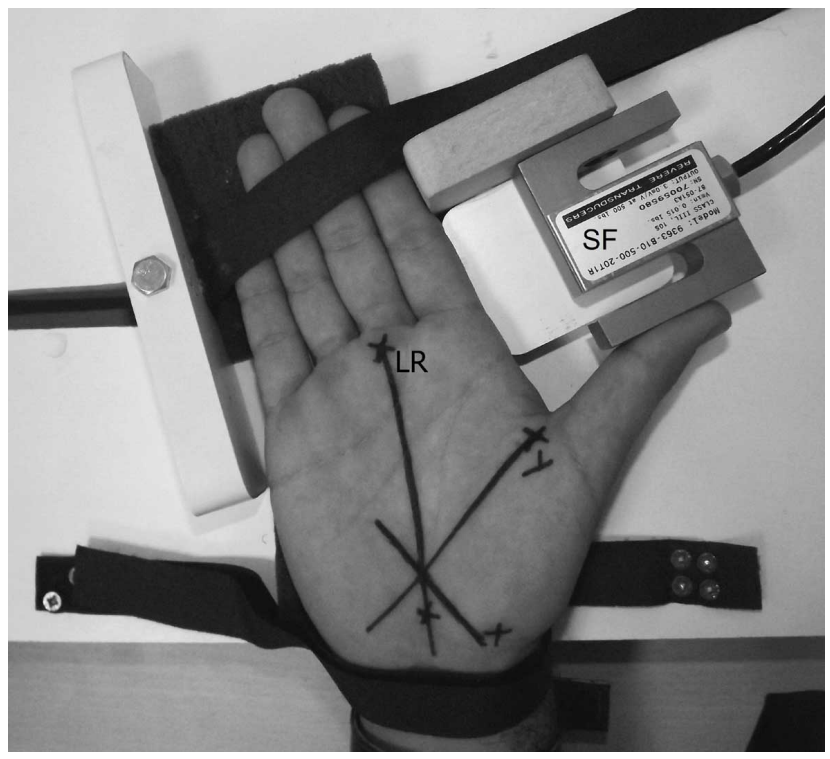

Fig. 2. Estructura de fijación de mano y pulgar. Nótese el sistema de referencia construido en la palma de la mano. LR: Línea de referencia, SF: Sensor de fuerza.

cado en una estructura de madera (Fig. 2). Esta estructura se utilizó para controlar y fijar la posición de la mano y del pulgar. Las fijaciones de ésta pudieron ser ajustadas a las características antropométricas de cada voluntario.

Procedimiento. Cada participante fue evaluado en posición sedente sobre una silla fijada al suelo, la espalda de los voluntarios estuvo totalmente apoyada en el respaldo de la silla, su brazo derecho pegado al tronco y su codo en flexión de $90^{\circ}$. La mano derecha fue fijada en posición supina en la estructura de madera, de tal forma que el vértice del sensor de fuerza coincidiera en el extremo más distal y medial de la falange proximal del pulgar (Fig. 2). 
Mediante el sensor de fuerza, se valoró la fuerza isométrica máxima voluntaria en el sentido de la flexión del pulgar. Se registraron tres repeticiones de cinco segundos de duración con un período de descanso de un minuto entre cada una de ellas. Se definió como fuerza voluntaria máxima (FVM) al máximo valor de fuerza registrado durante las tres contracciones evaluadas.

Una vez valorada la FVM, la piel sobre el FCP fue limpiada con una crema abrasiva (Everi, Spes Medica, Italia), con la finalidad de disminuir la impedancia eléctrica de la piel, y así mejorar la calidad de las señales EMGs. Luego, mediante un lápiz dermográfico e hipoalergénico se marcó un sistema de referencia de dos ejes (X, Y), con el objetivo de describir las ubicaciones de las ZI con respecto a este sistema de referencia (Fig. 2). Para la construcción de este sistema en la mano de cada voluntario, primero se trazó una línea de referencia (LR) desde el vértice palpable del extremo proximal de la primera falange del tercer dedo, hasta su intersección con el pliegue longitudinal radial (Fig. 2). El eje "Y" del sistema de referencia se dispuso desde el extremo palpable más distal y medial de la interlínea articular metacarpofalángica del pulgar, hasta su intersección con la LR. La dirección del eje "Y", se asumió paralela a las fibras del fascículo superficial del FCP. El eje "X" del sistema de referencia fue considerado como una línea perpendicular al eje "Y" y cuyo origen fue el punto de intersección entre el eje "Y" y la LR (Fig. 2). Los valores positivos del eje " $\mathrm{X}$ ", fueron considerados en dirección céfalo-lateral y para el eje "Y" caudo-lateral (Fig. 2).

Se ubicaron tres zonas de inervación (ZI) del FCP para cada voluntario evaluado. Estas ZI fueron determinadas para tres diferentes posiciones (ZI1, ZI2 y ZI3) del arreglo de 16 electrodos, el cual se ubicó siempre paralelo al eje "Y", a tres distancias de éste $(0,5$ y $10 \mathrm{~mm})$. La distancia de $10 \mathrm{~mm}$ fue fijada en base a la medición con resonancia nuclear magnética del ancho superficial del FCP en uno de los participantes de la muestra, la cual arrojó un ancho de 13,4mm (Fig. 3). Para localizar cada una de las ZI, se solicitó a los participantes mantener por 20 segundos, una contracción voluntaria isométrica en el sentido de la flexión del pulgar equivalente al 10\% de FVM. Los voluntarios recibieron la retroalimentación del nivel de fuerza generado mediante la visualización de este registro en un monitor ubicando frente a ellos. Durante cada contracción se registró la actividad EMGs del FCP. Las ZI fueron detectadas mediante la inversión de la dirección de propagación de los PAUM registrados a lo largo del arreglo de electrodos (Guzmán et al., 2011; Merletti et al., 2003; Merletti et al., 2008), tal como se muestra en la figura 4. Una vez localizadas las ZI, éstas fueron marcadas en la piel con un lápiz dermográfico, considerando las impresiones transitorias sobre la piel, que dejó el arreglo de electrodos (Figs. 3 y 4). La posi-

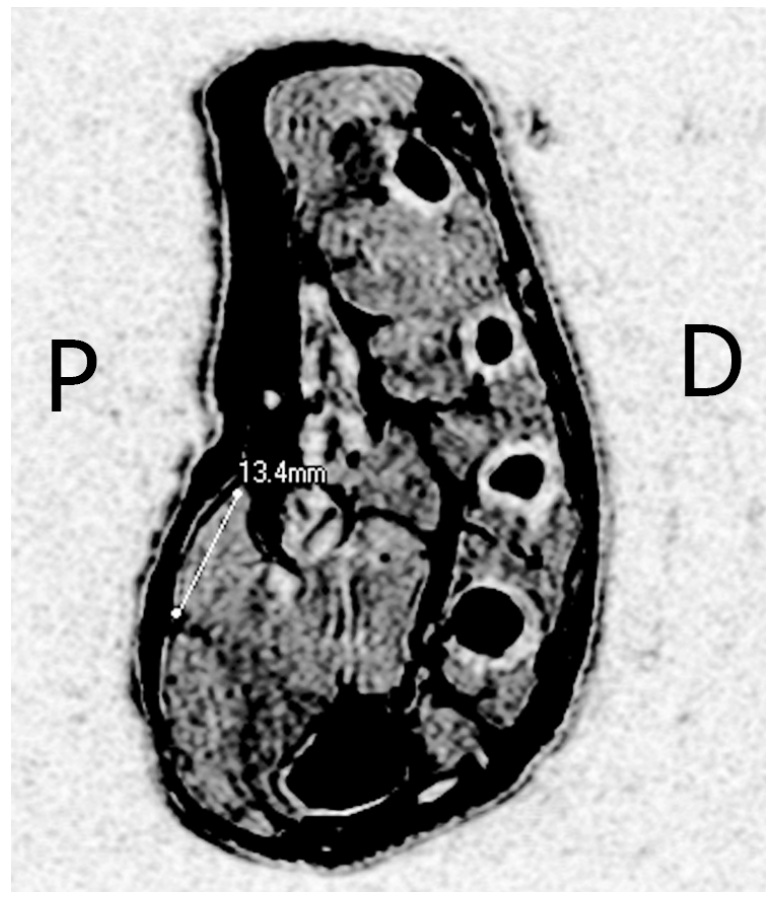

Fig. 3. Imágen de resonancia nuclear magnética en un corte trasversal de la mano derecha. Se muestra el ancho de 13.4 mm del músculo flexor corto del pulgar. P: palmar, D: dorsal.

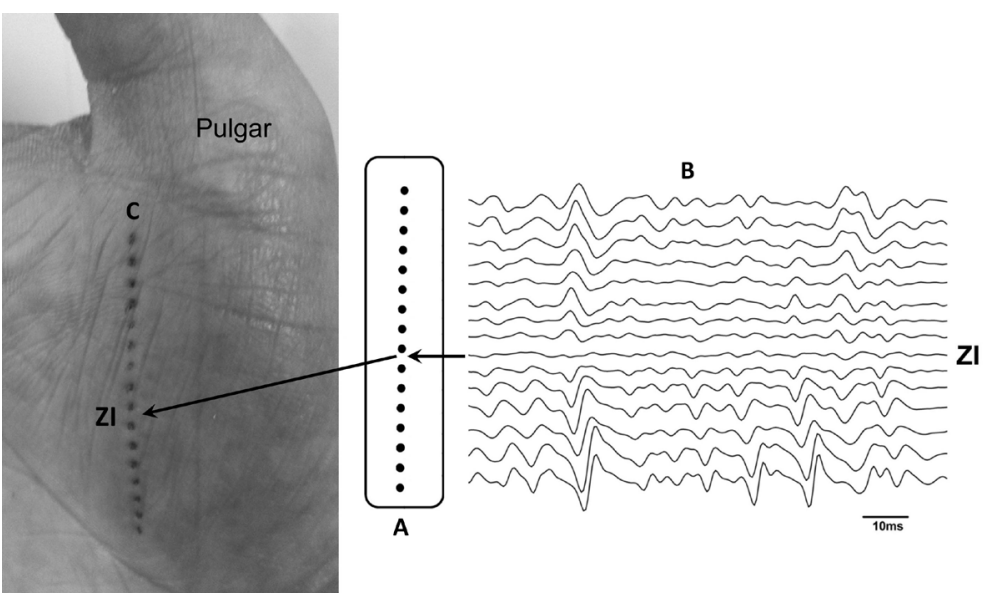

Fig. 4. Esquema de la localización de la ZI para una posición del arreglo de 16 electrodos. C: impresiones transitorias en la piel dejada por el arreglo de 16 electrodos (A). B: potenciales de acción de unidades motoras del flexor corto del pulgar, nótese el lugar de inversión de los potenciales, sitio que corresponde a la zona de inervación. 
ción de las ZI fueron expresadas como pares de coordenadas (X, Y), de las cuales las coordenadas en el sentido de "X" siempre fueron 0, 5 o $10 \mathrm{~mm}$, para ZI1, ZI2 y ZI3, respectivamente. Las coordenadas absolutas en "Y" de las ZI1, ZI2 y ZI3 fueron las distancias desde el extremo del eje "Y" hasta la ubicación de éstas, en sentido céfalo-medial. Con la intensión de descartar la influencia de las variaciones antropométricas de la muestra, las coordenadas en "Y" de las ZI de cada participante fueron ajustadas a la longitud total del eje "Y" de cada uno de ellos, y expresadas como porcentajes de dicha magnitud. Éstas últimas fueron denominadas como coordenadas relativas de las ZI.

Análisis estadístico. Para alcanzar el objetivo principal de este estudio se realizó una estadística descriptiva de las ubicaciones de las ZI (tanto en sus datos absolutos como relativos). Este mismo tipo de análisis fue aplicado a las características demográficas de la muestra. Por otro lado, se examinó el tipo de distribución de los datos aplicando la prueba de Shapiro Willk. Para cumplir con el objetivo secundario se aplicó una estática comparativa utilizando una prueba estadística de ANOVA y post prueba de Tukey. El nivel de confianza seleccionado para los análisis fue de un $95 \%$. Todos los procedimientos estadísticos fueron realizados en un software especializado en bioestadística (Prism 5.0. GraphPad Software, Inc).

\section{RESULTADOS}

Los datos son presentados en sus valores promedios \pm sus desviaciones estándares. En la tabla I se presentan las características demográficas de la muestra. Al comparar los datos demográficos entre los grupos, se observó una diferencia significativa entre los grupos en relación a la talla (promedio de diferencia: $-13,07 ; \mathrm{q}=7,74 ; \mathrm{p}<0,0001$ ) y la masa (promedio de diferencia: $-12,60 ; \mathrm{q}=7,46 ; \mathrm{p}<0,0001$ ). Mientras que no existió diferencia significativa en la edad (promedio de diferencia: $-1,67$; $q=0,98 ; \mathrm{p}>0,05)$. Las ubicaciones absolutas y relativas de las ZI1, ZI2 y ZI3 en ambos grupos tuvieron distribuciones normales (Shapiro Wilk, $\mathrm{p}>0,05)$. Al comparar las posiciones de las ZI al interior de cada grupo y entre los grupos, no se observaron diferencias significativas, $(\mathrm{p}>0,05)$ tanto para las posiciones absolutas como relativas. Los promedios de las posiciones absolutas y relativas para ambos grupos son mostradas en la Tabla II y en la figura 5, respectivamente. Considerando que no existió diferencia significativa en las posiciones de ambos grupos, se promediaron las ubicaciones de las ZI de los dos grupos, con la finalidad de obtener un valor representativo de la ubicación de la ZI del FCP para toda la muestra. Para la ubicación relativa, el promedio fue de $41,9 \pm 8.1 \%$ (Inter-

Tabla I. Características demográfica de la muestra. Promedio \pm desviación estándar.

\begin{tabular}{cccc}
\hline Grupos & Edad (años) & Peso $(\mathrm{kg})$ & Altura $(\mathrm{cm})$ \\
\hline Hombres $(\mathrm{n}=15)$ & $21,5 \pm 2,6$ & $70,7 \pm 7,2^{*}$ & $175,0 \pm 5,5^{*}$ \\
Mujeres $(\mathrm{n}=15)$ & $19,9 \pm 1,4$ & $57,9 \pm 11,1^{*}$ & $161,9 \pm 6,6^{\#}$ \\
\hline
\end{tabular}

*,\# Diferencia estadística $\mathrm{p}<0.05$.

Tabla II. Localización absoluta de las ZI. Promedio \pm desviación estándar.

\begin{tabular}{lccc}
\cline { 2 - 4 } Grupos & \multicolumn{3}{c}{ Localización (mm) } \\
\cline { 2 - 4 } & ZI1 & ZI2 & ZI3 \\
\hline Hombres $(\mathrm{n}=15)$ & $23 \pm 4$ & $23 \pm 5$ & $24 \pm 6$ \\
Mujeres $(\mathrm{n}=15)$ & $20 \pm 4$ & $20 \pm 5$ & $21 \pm 5$ \\
\hline
\end{tabular}

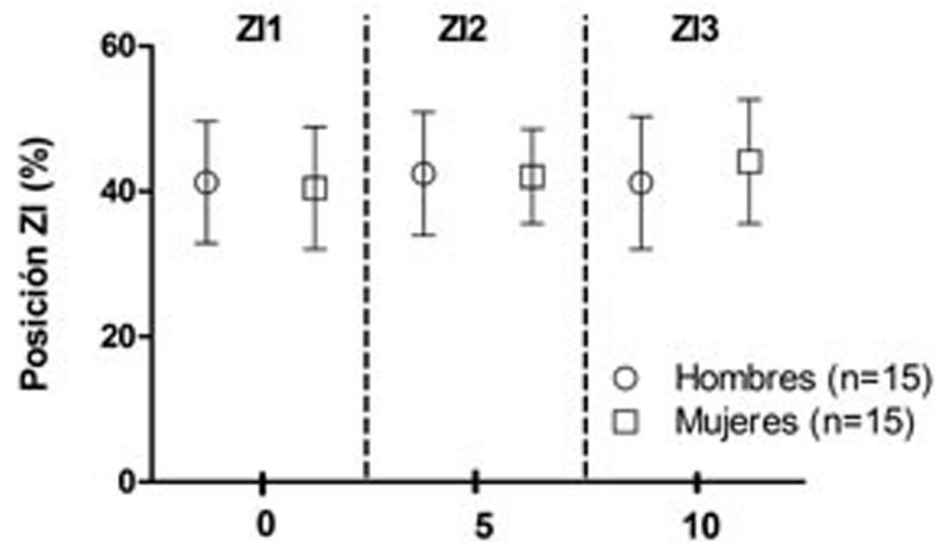

Posición X(mm)
Fig. 5. Promedios y desviaciones estándar de las ubicaciones relativas de las zonas de inervación del flexor corto del pulgar en ambos grupos de estudios. No se observó diferencia significativa al interior de cada grupo y entre los grupos. $(\mathrm{p}<0.05)$ 
valo inferior de confianza del promedio al 95\% inferior: 40,18\%; Intervalo superior de confianza del promedio al 95\%: 43,59\%; Coeficiente de variación 19,42\%). Mientras que para la posición absoluta fue de 22,0 $\pm 4.8 \mathrm{~mm}$ (Intervalo inferior de confianza del promedio al 95\% inferior: $21,02 \%$; Intervalo superior de confianza del promedio al 95\%: 23,05\%; Coeficiente de variación 21,96\%).

\section{DISCUSIÓN}

Las ZI corresponden a la ubicación de las placas motoras que forman parte de las unidades motoras de un músculo. Dada las características estructurales de la placa motora, su ubicación relativa a la fibra muscular permanece constante a pesar que la longitud de la fibra muscular pueda variar. Sin embargo, al momento de describir dicha ubicación en relación a referencias anatómicas externas al músculo, como prominencias óseas o pliegues cutáneos - tal como fue realizado en este estudio y habitualmente aplicado en la práctica clínica - ésta ubicación puede ser afectada por la posición articular y/o el nivel de fuerza generado por el músculo estudiado. Estas dos variables inciden directamente en la longitud de la fibra muscular, lo cual afecta la ubicación de la ZI en relación a referencias distintas a la fibra muscular. (DeFreitas et al., 2008; DeFreitas et al., 2010; Piitulainen et al., 2009). En el presente estudio la posición articular y el nivel de fuerza muscular fueron controlados. La posición del pulgar fue estandarizada fijando la mano y el pulgar mediante la estructura diseñada especialmente para ello. El nivel de fuerza solicitado durante el procedimiento de identificación de las ZI, fue controlado mediante la retroalimentación del registro del sensor de fuerza. De esta forma se logró que todos los participantes realizaran una contracción voluntaria en el sentido de la flexión del pulgar al mismo nivel porcentual de sus fuerzas máximas, con ello se pretendió que todos experimentaran una variación de longitud muscular muy similar, de esta forma homogenizar el desplazamiento de las ZI durante las contracciones voluntarias. En la localización de las ZI, se solicitó a los participantes realizar un muy bajo nivel de fuerza muscular $(10 \%$ FMV), con la finalidad de identificar la ZI a una longitud muscular muy cercana a su posición de reposo, lo que podría facilitar la ubicación de la ZI en el FCP en el ambiente clínico, haciendo este procedimiento algo independiente del registro de fuerza.

Adicionalmente las ubicaciones de las ZI en sentido del eje "Y", fueron normalizadas a la longitud de este eje, con la finalidad de eliminar los sesgos en la ubicación de la ZI relacionada con las diferencias antropométricas de los participantes. En el sentido del eje "X" las ubicaciones de las ZI no fueron normalizadas, dado que el ancho del músculo FCP es pequeño, estando al alrededor de unos 10$15 \mathrm{~mm}$. Este ancho fue corroborado mediante el registro de resonancia nuclear magnética realizado en uno de los voluntarios de la muestra. En base a ello, se decidió desplazar el arreglo de electrodos como máximo a 10mm del eje "Y", con la intensión de no registrar los potenciales que podrían generarse en el abductor corto del pulgar.

En la muestra evaluada no existieron diferencias en las ubicaciones absolutas y relativas de las ZI del FCP entre hombres y mujeres. Nuestros resultados demostraron que el sexo no tiene influencia en la distribución de las ZI en el FCP, por ende, en la ubicación de las placas motoras de las fibras musculares de dicho músculo. Por este motivo, los datos presentados en este estudio acerca de la ubicación relativa y absoluta de las ZI del FCP son aplicables tanto a hombres como a mujeres de la muestra evaluada.

\section{CONCLUSIÓN}

Mediante la EMGs, es posible identificar las ZI del FCP mediante un procedimiento no invasivo. No existen diferencias en la ubicación de las ZI entre los participantes de sexo masculino y femenino de la muestra evaluada. Para el grupo de participantes en este estudio, las ZI del FCP se encontraron en promedio al $41,9 \%$ de la distancia comprendida entre el extremo palpable más distal y medial de la interlínea articular metacarpofalángica del pulgar y una la línea que nace en el vértice palpable del extremo proximal de la primera falange del tercer dedo pasado por el pliegue longitudinal radial.

AGRADECIMIENTOS. Por su ayuda en la recolección de datos los autores agradecen a Florencia Mujica, Josefina Puntí y Sofía Contreras. Agradecemos especialmente al Dr. Julio Rosales Lecaros médico radiólogo de clínica MEDS, por su ayuda prestada en la obtención de los registros de resonancia nuclear magnética.

GUZMÁN, R.A.; BRALIC, E. M. P. \& CORDERO, G. J. Flexor pollicis brevis muscle innervation zone location in healthy individuals of both sexes. Int. J. Morphol., 31(2):449-454, 2013.

SUMMARY: The flexor pollicis brevis (FPB) is a muscle of the thenar eminence that plays an important role in thumb function. There is data about its innervation zone (IZ) distribution and sex differences. Knowing the location of the ZI of FPB could be helpful in treating spastic hand, serving to define the site of injection of the botulinum toxin. The aim of this study was to des- 
cribe the IZ location in the FPB using surface electromyography (sEMG), and also make a comparison between male and female subjects. Thirty young healthy volunteers participated in this study (15 males: $21.5 \pm 2.6$ years, $70.7 \pm 7.2 \mathrm{~kg}$ y $175.0 \pm 5.5 \mathrm{~cm} .15$ Females: $19.9 \pm 1.4$ years, $57.9 \pm 11.1 \mathrm{~kg}$ y $161.9 \pm 6.6 \mathrm{~cm})$. The IZ was identified by recording the action potentials of the FPB motor units, using a sixteen-electrode array. The action potentials were recorded during isometric contractions at $10 \%$ of maximum voluntary contraction. The location of the IZ was expressed as absolute and relative values in relation to a reference system constructed in the palm of the hand, based on anatomic references. There were not significant differences in the location of the IZ between male and female subjects. Of all the subjects, the IZ of the FPB was found at the $41.9 \%$ of the distance between the most distal and medial palpable extreme of the metacarpophalangeal joint line of the thumb and the line which rises at the palpable apex of the proximal side of the first phalanx of the third finger passing through the radial longitudinal fold.

Key Words: Motor Unit; Innervation zone; Motor Point; Flexor pollicis brevis.

\section{REFERENCIAS BIBLIOGRÁFICAS}

Danion, F. \& Gallea, C. The relation between force magnitude, force steadiness, and muscle co-contraction in the thumb during precision grip. Neurosci. Lett., 368(2):176-80, 2004.

Defreitas, J. M.; Costa, P. B.; Ryan, E. D.; Herda, T. J.; Cramer, J. T. \& Beck, T. W. An examination of innervation zone movement with increases in isometric torque production. Clin. Neurophysiol., 119(12):2795-9, 2008.

DeFreitas, J. M.; Costa, P. B.; Ryan, E. D.; Herda, T. J.; Cramer, J. T. \& Beck, T. W. Innervation zone location of the biceps brachii, a comparison between genders and correlation with anthropometric measurements. J. Electromyogr. Kinesiol., 20(1):76-80, 2010.

Gupta, S. \& Michelsen-Jost, H. Anatomy and function of the thenar muscles. Hand. Clin., 28(1):1-7, 2012.

Guzmán, R. A.; Silvestre, R. A. \& Arriagada, D. A. Ubicación de la zona de inervación del músculo bíceps braquial en sujetos sanos mediante electromiografía de superficie de alta densidad. Int. J. Morphol., 29(2):347-52, 2011.

Lapatki, B. G.; van Dijk, J. P.; van de Warrenburg, B. P. \& Zwarts, M. J. Botulinum toxin has an increased effect when targeted toward the muscle's endplate zone: a high-density surface EMG guided study. Clin. Neurophysiol., 122(8):1611-6, 2011.

Merletti, R.; Farina, D. \& Gazzoni, M. The linear electrode array: a useful tool with many applications. J. Electromyogr. Kinesiol., 13(1):37-47, 2003.
Merletti, R.; Holobar, A. \& Farina, D. Analysis of motor units with high-density surface electromyography. J. Electromyogr. Kinesiol., 18(6): 879-90, 2008.

Piitulainen, H.; Rantalainen, T.; Linnamo, V.; Komi, P. \& Avela, J. Innervation zone shift at different levels of isometric contraction in the biceps brachii muscle. J. Electromyogr. Kinesiol., 19(4):667-75, 2009.

Rainoldi, A.; Gazzoni, M. \& Casale, R. Surface EMG signal alterations in Carpal Tunnel syndrome: a pilot study. Eur. J. Appl. Physiol., 103(2):233-42, 2008.

Saitou, K.; Masuda, T.; Michikami, D.; Kojima, R. \& Okada, M. Innervation zones of the upper and lower limb muscles estimated by using multichannel surface EMG. J. Hum. Ergol., 29(1-2):35-52, 2000.

Dirección para correspondencia:

Rodrigo A. Guzmán PT, Msc.

Laboratorio Integrativo de Biomecánica y Fisiología del Esfuerzo (LIBFE)

Escuela de Kinesiología

Facultad de Medicina

Universidad de los Andes

Álvaro del Portillo 12455

Las Condes

Santiago - CHILE

Email: rguzman@uandes.cl

Recibido : 27-09-2012

Aceptado: 25-02-2013 